\title{
ARGÜIÇÕES DE DISSERTAÇÃO E DEFESAS DE TESE NOS ANOS
}

DE 1993 A 1994

1993

\section{DIREITO CIVIL}

Tese

: "Da desconstituição do vínculo filial (no Direito Brasileiro)"

Candidato

: José Luiz Gavião de Almeida

Orientador

: Álvaro Villaça Azevedo

Data da defesa

: 12.08.1993

Tese

: "Da eviç̧ão na compra e venda imobiliária"

Candidato

: Dorval Baptista Dallagnolo

Orientador

: Rubens Limongi França

Data da defesa

:04.10.1993

Dissertação

: "Um estudo sobre a indignidade no Direito Sucessório Brasileiro"

Candidato : Marcelo Fortes Barbosa Filho

Orientador : Yussef Said Cahali

Data da argüição : 12.11.1993

Tese : "O elemento subjetivo nas fraudes contra credores (aspectos fundamentais do consilium fraudis)"

Candidato : Paulo Roberto Tavares Paes

Orientador : Rubens Limongi França

Data da defesa $\quad: 29.11 .1993$

Tese : "Inseminação artificial"

Candidata . Elizabeth Alves de Freitas

Orientador Álvaro Villaça Azevedo

Data da defesa : 02.12 .1993 


\section{DIREITO COMERCIAL}

\begin{tabular}{|c|c|}
\hline Dissertação & : "Alienação do poder de controle acionário" \\
\hline Candidato & : Guilherme Doring Cunha Pereira \\
\hline Orientador & : Fábio Konder Comparato \\
\hline Data da argüição & $: 25.05 .1993$ \\
\hline Tese & $\begin{array}{l}\text { : "A tutela de interesses coletivos na Lei de Sociedade por } \\
\text { Ações" }\end{array}$ \\
\hline Candidato & Fernando Netto Boiteux \\
\hline Orientador & : Fábio Konder Comparato \\
\hline Data da defesa & 27.10 .1993 \\
\hline Tese & $\begin{array}{l}\text { : "O Conselho de Administração na sociedade anônima } \\
\text { brasileira: proposta ara sua melhor utilização" }\end{array}$ \\
\hline Candidato & : Paulo Fernando Campos Salles de Toledo \\
\hline Orientador & : Fábio Konder Comparato \\
\hline Data da defesa & : 09.12.1993 \\
\hline
\end{tabular}

DIREITO DO TRABALHO

Tese : "O acidente de trabalho e a Constituição de 1988: o futuro da questão acidentária"

Candidato Anníbal Fernandes

Orientador : Pedro Vidal Neto

Data da defesa $\quad: 10.02 .1993$

Dissertação : : juiz classista no Poder Judiciário. Uma forma de participação popular na administração da Justiça"

Candidato José Calheiros Ribeiro Ferreira

Orientador : Pedro Vidal Neto

Data da argüição : 11.05.1993 
Dissertação "De bóias-frias a cortadores de cana: o direito ao progresso com desordem na região de Ribeirão Preto"

Candidata Mayla Yara Porto

Orientador : Pedro Vidal Neto

Data da argüição : 02.06.1993

Dissertação : : "Arbitragem facultativa como solução dos conflitos do trabalho"

Candidata : Elisabeth Cavini

Orientador : Octávio Bueno Magano

Data da argüição : 25.08.1993

Dissertação : "A legitimidade para agir dos sindicatos no processo do trabalho"

Candidata : Regina Maria de Oliveira Vasconcelos

Orientador : Cássio de Mesquita Barros Júnior

Data da argüição $\quad 14.12 .1993$

\section{DIREITO DO ESTADO}

Dissertação : : "Iniciativa popular na Constituição de 1988"

Candidato . Hélcio Ribeiro

Orientador . Enrique Ricardo Lewandowski

Data da argüição : 31.05 .1993

Dissertação : : "O princípio da subsidiariedade no Direito Administrativo"

Candidato : Fernando Pimentel Cintra

Orientadora : Maria Sylvia Zanella Di Pietro

Data da argüição : 04.06.1993

Dissertação "As inovações constitucionais no Direito de Família"

Candidata Mirian Freire Pereira

Orientador : Enrique Ricardo Lewandowski

Data da argüição : 21.06 .1993 
Dissertação : "Terras indígenas no Brasil: uma contribuição para seu estudo"

Candidato : Marco Antônio Barbosa

Orientador : Dalmo de Abreu Dallari

Data da argüição : 09.08.1993

Tese : "O serviço público na atualidade"

Candidato : Marcelo Pereira

Orientadora : Odete Medauar

Data da defesa $\quad: 13.08 .1993$

Tese : "Parâmetros da notória especialização"

Candidato : Sergio Resende de Barros

Orientador Manoel Gonçalves Ferreira Filho

Data da defesa $\quad: 14.10 .1993$

Tese : "O controle de constitucionalidade por via de ação"

Candidato : Dircêo Torrecillas Ramos

Orientador Manoel Gonçalves Ferreira Filho

Data da defesa $\quad: 18.10 .1993$

DIREITO PENAL

Dissertação : : "O Direito Penal e a proteção do meio ambiente"

Candidata Gilda Pereira de Carvalho

Orientadora : Ivette Senise Ferreira

Data da argüição : 25.03.1993

Dissertação : : "Tipicidade e princípio da insignificância"

Candidato : Carlos Vico Mañas

Orientador Antonio Luis Chaves Camargo

Data da argüição : : 19.04.1993 


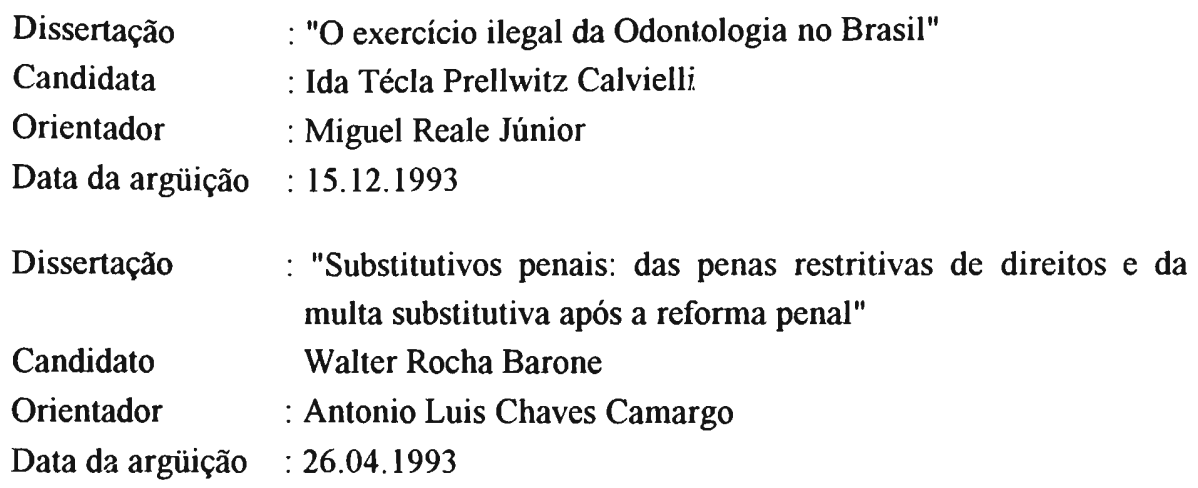

\section{DIREITO PROCESSUAL}

Dissertação : "Legitimidade passiva e autoridade coatora em mandado de segurança"

Candidato : Carlos Augusto de Assis

Orientador : Kazuo Watanabe

Data da argüição $\quad 23.04 .1993$

Dissertação "Ação de nunciação de obra nova"

Candidato Jorge Cocicov

Orientador : Vicente Greco Filho

Data da argüição : 28.04.1993

Dissertação : "Aplicação dos princípios constitucionais do processo no Direito Tributário"

Candidato : José Fernando Cedeño de Barros

Orientador Ada Pellegrini Grinover

Data da argüição : 01.06.1993

Dissertação : : "Aspectos processuais da Lei 8.069/90: o Estatuto da Criança e do Adolescente"

Candidata $\quad:$ Cristina de Freitas Cirenza

Orientador Luiz Carlos de Azevedo

Data da argüição $\quad 03.06 .1993$ 


$\begin{array}{ll}\text { Dissertação } & \text { : "O ônus da prova" } \\ \text { Candidata } & \text { : Cecília Matos } \\ \text { Orientador } & : \text { Kazuo Watanabe } \\ \text { Data da argüição } & : 27.06 .1993 \\ & \\ \text { Dissertação } & : \text { "Ação penal condenatória" } \\ \text { Candidata } & \text { : Paula Bager Fernandes } \\ \text { Orientador } & : \text { Sérgio Marcos de Moraes Pitombo } \\ \text { Data da argüição } & : 25.06 .1993\end{array}$

Dissertação : : "O duplo grau de jurisdição no Direito Processual Civil"

Candidato : Oreste Nestor de Souza Laspro

Orientador : José Rogério Cruz e Tucci

Data da argüição : 03.11 .1993

Dissertação : "As garantias da liberdade individual no Código de Processo Penal Brasileiro"

Candidato : Fernando Pavan Baptista

Orientador . Rogério Lauria Tucci

Data da argüição : 24.11 .1993

Dissertação : "Assistência jurídica, assistência judiciária e justiça gratuita"

Candidato Augusto Tavares Rosa Marcacini

Orientador : Luiz Carlos de Azevedo

Data da argüição : 19.11.1993

Dissertação "O valor da causa no Direito Processual Civil Brasileiro"

Candidata : Marina Mesquita

Orientador : Luiz Carlos Azevedo

Data da argüição : 30.11 .1993 


\section{DIREITO ECONÔMICO-FINANCEIRO}

Dissertação : "A questão ambiental e o Direito Econômico"

Candidata Fátima Cristina Bonassa Bucker

Orientador : Fábio Nusdeo

Data da argüição : 08.03.1993

Dissertação : : "Isenção tributária"

Candidato : Chan Tzu Yao

Orientador Paulo Roberto Cabral Nogueira

Data da argüição : 14.05.1993

Dissertação "Direito de planejamento econômico e social"

Candidato : Carlos Shiro Takahashi

Orientador : Fábio Nusdeo

Data da argüição : 17.05.1993

Tese : "Planejamento fiscal através de acordos de bitributação: treaty shopping"

Candidato : : Luis Eduardo Schoueri

Orientador : Gerd Willi Rothmann

Data da defesa $\quad: 30.06 .1993$

Dissertação : :A conversão da dívida externa brasileira em investimento"

Candidato : José Luis Conrado Vieira

Orientador : Fábio Nusdeo

Data da argüição $\quad 02.07 .1993$

Dissertação : : "Contribuição ao estudo de controle de preços"

Candidato : Fábio Pugliesi

Orientador : Eros Roberto Grau

Data da argüição : 30.08 .1993 


\section{DIREITO INTERNACIONAL}

Dissertação : : "Princípios de Relações Exteriores da Constituição Brasileira de 1988"

Candidato : Pedro Bohomoletz de Abreu Dallari

Orientador Celso Lafer

Data da argüição $\quad 05.03 .1993$

Dissertação : "O comércio internacional de serviços na perspectiva do Direito Internacional Econômico"

Candidata : Valéria da Silva Nunes

Orientador : Luis Olavo Baptista

Data da argüição : 16.04.1993

Dissertação -European Community Investiment Partner: o Direito regulamentando a cooperação internacional"

Candidata : Suzana Camargo Vieira

Orientador : Celso Lafer

Data da argüição : 10.05.1993

Dissertação : "Da guerra justa (Um estudo jurídico-filosófico da teoria do Bellum Justum nas concepções decisionista e intelectual e na síntese grociana)"

Candidato : Hilton Catanzaro Guimarães

Orientador : José Roberto Franco da Fonseca

Data da argüição : 23.06.1993

Dissertação "A evolução recente dos processos de integração em LatinoAmérica: a formação de blocos, com especial referência ao Grupo dos Três constituído por México, Colômbia e Venezuela e Centro-América"

Candidata : Eneyda Terezita Madriz Patiño

Orientador : José Roberto Franco da Fonseca

Data da argüição : 05.10.1993 
Tese

\section{FILOSOFLA E TEORIA GERAL DO DIREITO}

Dissertação : : "Direito - burocracia e práxis: um ensaio crítico epistemológico"

Candidato : Luiz Antonio Lucena de Oliva

Orientador : Aloysio Ferraz Pereira

Data da argüição : 09.06.1993

Dissertação : : :O abuso do direito de propriedade no Direito Romano Clássico"

Candidato : José Luiz Levy

Orientador : Alexandre Augusto de Castro Corrêa

Data da argüição : 18.06.1993

Dissertação "Direito, legitimação e disciplina: intersecção Foucault e Luhmann"

Candidato : Dácio Girardi

Orientador : José Eduardo Campos de Oliveira Faria

Data da argüição $\quad 06.08 .1993$

Tese : "O surgimento do ordenamento jurídico capitalista no Brasil"

Candidato Everaldo Tadeu Quilici Gonzalez

Orientador : Aloysio Ferraz Pereira

Data da defesa $\quad: 25.08 .1993$ 


\section{DIREITO CIVIL}

\begin{tabular}{|c|c|}
\hline Dissertação & : "Cessão de herança" \\
\hline Candidato & : Evadren Antonio Flaiban \\
\hline Orientador & : Daisy Gogliano \\
\hline Data da defesa & : 10.05 .94 \\
\hline Dissertação & : "Usucapião agrário" \\
\hline Candidato & Antonio Augusto de Souza Coelho \\
\hline Orientador & : Giselda Maria Fernandes Novaes Hironaka \\
\hline Data da defesa & $: 24.05 .94$ \\
\hline Dissertação & $\begin{array}{l}\text { : "Direito à honra: delineamentos e âmbito da tutela no Direito } \\
\text { Brasileiro Hodierno" }\end{array}$ \\
\hline Candidato & : José D'Amico Bauab \\
\hline Orientador & : Carlos Alberto Bittar \\
\hline Data da defesa & $: 06.06 .94$ \\
\hline Dissertação & $\begin{array}{l}\text { : "O desenvolvimento agrícola sustentado: princípio do Direito } \\
\text { Agrário e sua aplicação penal" }\end{array}$ \\
\hline Candidato & : Paulo Sérgio Miguez Urbano \\
\hline Orientador & : Giselda Maria Fernandes Novaes Hironaka \\
\hline Data da defesa & 07.06 .94 \\
\hline Dissertação & $\begin{array}{l}\text { : "A tutela estatal de conteúdo civil e o Estatuto da Criança e do } \\
\text { Adolescente" }\end{array}$ \\
\hline Candidato & Sérgio Matheus Santos Garcez \\
\hline Orientador & Sérgio Carlos Covello \\
\hline Data da defesa & $: 09.06 .94$ \\
\hline
\end{tabular}


Tese

Candidato

Orientador

Data da defesa

Dissertação

Candidato

Orientador

Data da defesa

Tese

Candidato

Orientador

Data da defesa

Dissertação

Candidato

Orientador

Data da defesa
"A propriedade aparente (a aquisição a mon domino da propriedade imóvel com eficácia translativa no Código Civil" Francisco Antonio Paes Landim Filho

: Fábio Maria De-Mattia

: 10.08 .94

: "Separação de fato (efeitos pessoais e patrimoniais entre cônjuges)"

Sylvia Soares de Mello do Val

Rubens Limongi França

: 22.08.94

: "Exclusão do condômino nocivo no condomínio em edificios"

: Maria Regina Pagetti Moran

Álvaro Villaça Azevedo

30.08.94

: "O Direito Agrário e as terras indígenas - Um estudo comparado na América Latina"

Nancy Sgursky Lodeiro

: Fábio Maria De-Mattia

: 15.12 .94

\section{DIREITO COMERCIAL}

Dissertação : : "A responsabilidade dos administradores de Sociedades Limitadas"

Candidato : : Tony Tsuyoshi Kazama

Orientador : Vera Helena de Mello Franco

Data da defesa $\quad: 02.08 .94$ 
DIREITO DO TRABALHO

Dissertação "O direito adquirido à aposentadoria por tempo de serviço"

Candidato Domênico Antonio Landulfo

Orientador : Pedro Vidal Neto

Data da defesa : 30.05 .94

Dissertação : : "Interesses difusos no Direito do Trabalho"

Candidato : João Hilário Valentim

Orientador Oris de Oliveira

Data da defesa $\quad: 13.06 .94$

Dissertação : : "Direito Coletivo do Trabalho e os servidores públicos"

Candidato : Robertônio Santos Pessoa

Orientador : Oris de Oliveira

Data da defesa $\quad: 20.06 .94$

Tese "As transformações do Direito do Trabalho"

Candidato : : Luiz Carlos Amorim Robortella

Orientador : Octávio Bueno Magano

Data da defesa $: 23.06 .94$

Dissertação "A interpretação jurídica e as perspectivas do Direito do Trabalho"

Candidato : Suzete Carvalho

Orientador Amauri Mascaro Nascimento

Data da defesa $\quad 08.08 .94$

Dissertação : : "Novas dimensões da alteração do Contrato de Trabalho"

Candidato : Cassius Marcellus Zomignani

Orientador : Cássio de Mesquita Barros Junior

Data da defesa : 09.08 .94 
Dissertação : : "Uma proposta para reformulação do Processo do Trabalho: A criação de juizados de pequenas causas trabalhistas"

Candidato : Patricia Tuma Martins Bertolin

Orientador : Wagner Drdla Giglio

Data da defesa $: 31.08 .94$

Dissertação : : "Convenção coletiva de trabalho no Direito Brasileiro Setor Privado"

Candidato : Paulo Eduardo Vieira de Oliveira

Orientador Pedro Vidal Neto

Data da defesa $\quad 27.10 .94$

Dissertação : "A competência funcional do Juiz Presidente da Junta de Conciliação e Julgamento"

Candidato Manoel Carlos Toledo Filho

Orientador : Oris de Oliveira

Data da defesa : :07.11.94

DIREITO DO ESTADO

Tese : "Da competência em matéria administrativa"

Candidato : Massami Uyeda

Orientador : José Cretella Júnior

Data da defesa $\quad: 29.03 .94$

Dissertação "Considerações sobre a democracia e sua relação com sistemas de governo"

Candidato : Marcos Fábio de Oliveira Nusdeo

Orientador : Anna Cândida da Cunha Ferraz

Data da defesa $\quad: 16.05 .94$ 
Dissertação : : "Referendo e iniciativa popular na perspectiva dos modelos suíço e norte-americano"

Candidato : Sônia Boczar

Orientador : Manoel Gonçalves Ferreira Filho

Data da defesa $\quad: 14.06 .94$

Tese : :O desvio de finalidade nas licitações"

Candidato : Carlos Borges de Castro

Orientador : José Cretella Júnior

Data da defesa $\quad: 21.06 .94$

Dissertação : "A exceptio non adimpleti contractus (no âmbito do Direito Administrativo)"

Candidato : Jocélia de Almeida Castilho

Orientador José Cretella Júnior

Data da defesa $\quad: 24.06 .94$

Tese

Candidato

: "Privatização no Estado Contemporâneo"

Orientador : Odete Medauar

Data da defesa $\quad: 10.10 .94$

Dissertação : "Da mutabilidade dos contratos administrativos"

Candidato Antonieta Rosa Gomes

Orientador : Maria Sylvia Zanella Di Pietro

Data da defesa $\quad 30.11 .94$

Dissertação "Mandado de Segurança Coletivo"

Candidato : David Diniz Dantas

Orientador : Manoel Gonçalves Ferreira Filho

Data da defesa $\quad: 05.12 .94$ 
$\begin{array}{ll}\text { Dissertação } & \text { : "O Tribunal de Contas: competência, ação fiscalizadora e } \\ & \text { princípios norteadores" } \\ \text { Candidato } & : \text { Anna Hilda de Almeida Donadio } \\ \text { Orientador } & : \text { Enrique Ricardo Lewandowski } \\ \text { Data da defesa } & : 06.12 .94\end{array}$

DIREITO PENAL

Dissertação : : "Direito Subjetivo e antijuridicidade material"

Candidato : Paulo de Sena Martins

Orientador : Ivette Senise Ferreira

Data da defesa $\quad: 02.02 .94$

Dissertação : "A proteção penal ao patrimônio imóvel rural (Invasores rurais)"

Candidato Elisabete Maniglia

Orientador : Miguel Reale Júnior

Data da defesa $\quad: 06.12 .94$

Tese : "Crimes cometidos com uso de computador"

Candidato : : Pedro Luiz Ricardo Gagliardi

Orientador : Ivette Senise Ferreira

Data da defesa $\quad 16.12 .94$

DIREITO PROCESSUAL

Tese "Perfil histórico e evolução da perícia - Perícia cronológica de disparo de armas de fogo curtas, como elemento de prova"

Candidato : José Lopes Zarzuela

Orientador : José Rogério Cruz e Tucci

Data da defesa $\quad: 15.03 .94$ 
$\begin{array}{ll}\text { Dissertação } & : \text { "As garantias constitucionais na investigação criminal" } \\ \text { Candidato } & : \text { Fauzi Hassan Choukr } \\ \text { Orientador } & : \text { Ada Pellegrini Grinover } \\ \text { Data da defesa } & : 22.03 .94\end{array}$

Tese : "Tutela jurisdicional (A relativação do binômio direitoprocesso, como meio de acesso à ordem juridica justa"

Candidato : José Roberto dos Santos Bedaque

Orientador Cândido Rangel Dinamarco

Data da defesa $\quad 06.05 .94$

Dissertação : : : A técnica de elaboração da sentença civil"

Candidato : Nelton Agnaldo Moraes dos Santos

Orientador : Vicente Greco Filho

Data da defesa $\quad: 12.05 .94$

Dissertação "Contribuição ao estudo da fundamentação das decisões no Direito Processual"

Candidato Carlos Alberto da Costa Dias

Orientador : Vicente Greco Filho

Data da defesa $\quad: 19.05 .94$

Dissertação : : "Provas ilícitas"

Candidato : Luis Francisco Torquato Avolio

Orientador : Ada Pellegrini Grinover

Data da defesa $: 10.06 .94$

Tese $\quad:$ O processo de execução penal"

Candidato : Sidnei Agostinho Benetti

Orientador : Rogério Lauria Tucci

Data da defesa $\quad$ :08.07.94

Dissertação : : "A defesa do réu na história do processo"

Candidato : Luiz George Navarro

Orientador José Rogério Cruz e Tucci

Data da defesa $\quad 22.11 .94$ 


\section{DIREITO ECONÔMICO-FINANCEIRO}

\begin{tabular}{|c|c|}
\hline Dissertação & "O empréstimo compulsório no Direito Brasileiro" \\
\hline Candidato & : Marcos Simão Figueras \\
\hline Orientador & Walter Barbosa Corrêa \\
\hline Data da defesa & $: 15.06 .94$ \\
\hline Dissertação & $\begin{array}{l}\text { "O Direito Subjetivo, o concuso extra-judicial e a ordem } \\
\text { econômica" }\end{array}$ \\
\hline Candidato & Antonio Amílcar de Oliveira Lima \\
\hline Orientador & Eros Roberto Grau \\
\hline Data da defesa & : 30.06 .94 \\
\hline Dissertação & : "Inflação e Imposto de Renda da pessoa jurídica" \\
\hline Candidato & : João Francisco Bianco \\
\hline Orientador & Ruy Barbosa Nogueira \\
\hline Data da defesa & : 12.09 .94 \\
\hline
\end{tabular}

Dissertação : : "Os aspectos constitucionais da contribuição social sobre o lucro das pessoas jurídicas"

Candidato João Sampaio Rego Neto

Orientador : Ruy Barbosa Nogueira

Data da defesa $\quad: 20.10 .94$

Dissertação : "Disciplina jurídica da extrafiscalidade"

Candidato : Leila Paiva

Orientador : Gerd Willi Rothmann

Data da defesa $\quad: 04.11 .94$

Dissertação "Contribuições interventivas na nova ordem constitucional Um estudo"

Candidato : Artur Barbosa Parra

Orientador : Ruy Barbosa Nogueira

Data da defesa $\quad: 24.11 .94$ 


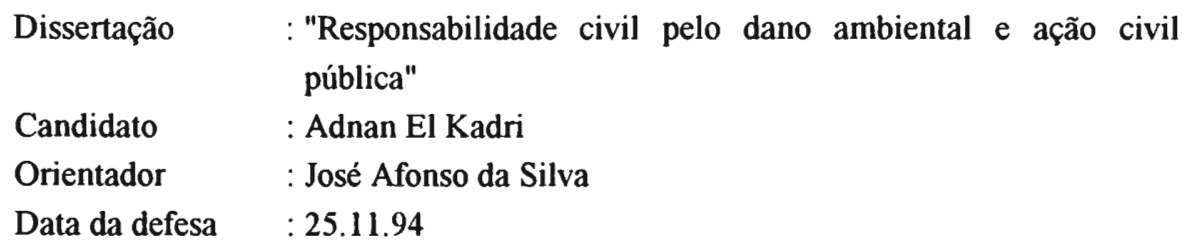

Dissertação "Aspectos juridicos do cooperativismo e das cooperativas de habitação no Brasil"

Candidato : Maria Paula Dallari Bucci

Orientador José Afonso da Silva

Data da defesa $\quad: 28.11 .94$

Dissertação : "A tributação da unidade familiar pelo Imposto de Renda"

Candidato : Rose Anna Augusto Mariano

Orientador : Alcides Jorge Costa

Data da defesa $\quad: 07.12 .94$

\section{DIREITO INTERNACIONAL}

Tese

Candidato

Orientador

Data da defesa

Tese

Candidato

Orientador

Data da defesa
"Negociação e formação dos contratos internacionais do comércio - Direito Comparado e prática dos negócios"

Maristela Basso

: Luiz Olavo Baptista

: 25.04 .94

: "Contribuição ao estudo dos contratos internacionais de knowhow"

: Luiz Alfredo Ribeiro da Silva Paulin

: Luiz Olavo Baptista

: 23.05.94 


\begin{tabular}{|c|c|}
\hline Dissertação & $\begin{array}{l}\text { : "Noções e instituições de Direito Internacional na Grécia } \\
\text { Antiga" }\end{array}$ \\
\hline Candidato & : José Blanes Sala \\
\hline Orientador & Guido Fernando Silva Soares \\
\hline Data da defesa & 27.05 .94 \\
\hline Dissertação & : "As concordatas e o Direito Internacional" \\
\hline Candidato & Antonio Benedito do Nascimento \\
\hline Orientador & : José Carlos de Magalhães \\
\hline Data da defesa & 26.08 .94 \\
\hline Dissertação & $\begin{array}{l}\text { :Os desafios do Direito Internacional em face de um problema } \\
\text { ambiental global: O efeito estufa" }\end{array}$ \\
\hline Candidato & Luciana Mancini \\
\hline Orientador & Luiz Olavo Baptista \\
\hline Data da defesa & : 02.09 .94 \\
\hline Dissertação & $\begin{array}{l}\text { : "Refugiados: Evolução de seu conceito e de sua instituição à } \\
\text { luz do Direito das Gentes" }\end{array}$ \\
\hline Candidato & José Henrique Fischel de Andrade \\
\hline Orientador & Celso Lafer \\
\hline Data da defesa & 19.09 .94 \\
\hline Dissertação & $\begin{array}{l}\text { : O contrato de transporte aéreo e a responsabilidade do } \\
\text { transportador" }\end{array}$ \\
\hline Candidato & Maria Esther Fernandez Alvarez \\
\hline Orientador & Irineu Strenger \\
\hline Data da defesa & 23.11 .94 \\
\hline
\end{tabular}

\section{FILOSOFLA E TEORIA GERAL DO DIREITO}

Dissertação : "Crítica da prudência e sua aplicação ao Direito"

Candidato : Mauro de Medeiros Keller

Orientador Aloysio Ferraz Pereira

Data da defesa $\quad: 24.06 .94$ 
Tese : : "Contribuição para um pensamento jurídico brasileiro"

Candidato : Jeannette Antonios Maman

Orientador : Aloysio Ferraz Pereira

Data da defesa $\quad: 15.08 .94$

Dissertação "Ensaio sobre Direito Subjetivo e situação jurídica"

Candidato Silma Marlice Sorub de Souza

Orientador : Maria Celeste Cordeiro Leite dos Santos

Data da defesa $\quad 14.12 .94$ 